\title{
Prevalence of fluoroquinolone resistance and mutations in the gyrA, parC and parE genes of Riemerella anatipestifer isolated from ducks in China
}

Dekang Zhu ${ }^{1,2+}$, Mingyu Zheng ${ }^{1,2 \dagger}$, Jinge Xu ${ }^{3}$, Mingshu Wang ${ }^{1,4,2}$, Renyong Jia ${ }^{1,4,2}$, Shun Chen ${ }^{1,4,2}$, Mafeng Liu ${ }^{1,4,2}$,

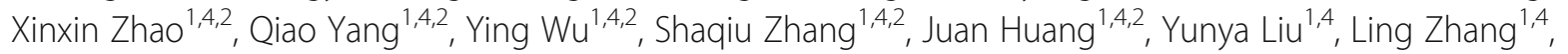
Yanling $\mathrm{Yu}^{1,4}$, Leichang Pan ${ }^{1,4}$, Xiaoyue Chen ${ }^{1,2}$ and Anchun Cheng ${ }^{1,4,2^{*}}$ (D)

\begin{abstract}
Background: Riemerella anatipestifer is one of the most serious infectious disease-causing pathogens in the duck industry. Drug administration is an important method for prevention and treatment of infection in duck production, leading to widespread drug resistance in $R$. anatipestifer.

Methods: For a total of 162 isolates of $R$. anatipestifer, the MICs were determined for a quinolone antimicrobial agent, namely, nalidixic acid, and three fluoroquinolones, namely, ciprofloxacin, enrofloxacin and ofloxacin. The gyrA, parC, and parE gene fragments were amplified by PCR to identify the mutation sites in these strains. Site-directed mutants with mutations that were detected at a high frequency in vivo were constructed (hereafter referred to as site-directed in vivo mutants), and the MICs of these four drugs for these strains were determined.
\end{abstract}

Results: In total, 100, 97.8, 99.3 and 97.8\% of the 137 R. anatipestifer strains isolated between 2013 and 2018 showed resistance to nalidixic acid, ciprofloxacin, enrofloxacin, and ofloxacin, respectively. The high-frequency mutation sites were detected in a total of 162 R. anatipestifer strains, such as Ser83lle and Ser83Arg, which are two types of substitution mutations of amino acid 83 in GyrA; Val799Ala and Ile811Val in ParC; and Val357lle, His358Tyr, and Arg541Lys in ParE. MIC analysis results for the site-directed in vivo mutants showed that the strains with only the Ser83lle mutation in GyrA exhibited an 8-16-fold increase in MIC values, and all mutants showed resistance to ampicillin and ceftiofur.

Conclusions: The resistance of $R$. anatipestifer to quinolone agents is a serious problem. Amino acid 83 in GyrA is the major target mutation site for the fluoroquinolone resistance mechanism of $R$. anatipestifer.

Keywords: Riemerella anatipestifer, Fluoroquinolone resistance, Point mutant, gyrA gene, parC gene, parE gene

\footnotetext{
* Correspondence: chenganchun@vip.163.com

${ }^{\dagger}$ Dekang Zhu and Mingyu Zheng contributed equally to this work.

${ }^{1}$ Research Center of Avian Diseases, College of Veterinary Medicine, Sichuan

Agricultural University, Chengdu, Sichuan, China

${ }^{4}$ Guizhou Animal Husbandry and Veterinary Research Institute, Guiyang,

Guizhou, China

Full list of author information is available at the end of the article
}

C The Author(s). 2019 Open Access This article is distributed under the terms of the Creative Commons Attribution 4.0 International License (http://creativecommons.org/licenses/by/4.0/), which permits unrestricted use, distribution, and reproduction in any medium, provided you give appropriate credit to the original author(s) and the source, provide a link to the Creative Commons license, and indicate if changes were made. The Creative Commons Public Domain Dedication waiver (http://creativecommons.org/publicdomain/zero/1.0/) applies to the data made available in this article, unless otherwise stated. 


\section{Background}

Riemerella anatipestifer ( $R$. anatipestifer) is a nonmotile Gram-negative rod-shaped bacterium that is usually isolated from ducks, geese, and turkeys. $R$. anatipestifer infection can cause pericarditis, peritonitis, fibrinous exudation, diarrhea and neurological symptoms in ducks, which lead to reduced growth rates and high mortality and consequently to great economic loss [1].

Fluoroquinolone antibiotics are one of the most potent broad-spectrum agents commonly used to treat a range of infections $[2,3]$. Due to the widespread and indiscriminate use of fluoroquinolone antibiotics, the resistance of $R$. anatipestifer to fluoroquinolones is particularly serious $[4,5]$.

Many types of antibiotic resistance mechanisms of $R$. anatipestifer have been reported, including those against chloramphenicol [6]; florfenicol [7]; aminoglycosides [8]; macrolides, lincosamides and streptogramin B (collectively referred to as MLS) [9, 10]; and tetracycline [11]. However, few articles on the mechanism of fluoroquinolone resistance associated with $R$. anatipestifer have been published. Resistance to quinolones can occur via multiple mechanisms, including target-site mutations, multidrug resistance (MDR) efflux pumps, changes in membrane permeability, and plasmid-mediated quinolone resistance (PMQR) genes [12].

DNA gyrase and topoisomerase IV can both relax positively supercoiled DNA, albeit with different focuses. DNA gyrase is essential for DNA replication, transcription, and recombination [12]. Topoisomerase IV unlinks newly replicated DNA, thereby allowing chromosome segregation at cell division [13]. Studies on target mutations in quinolone-resistance-determining regions (QRDRs) show that these mutations are the most prevalent mechanism of quinolone resistance. Mutations involved in fluoroquinolone resistance have been extensively studied in other species in recent decades, such as Escherichia coli [14], Mycobacterium tuberculosis [15], and Shigella flexneri [16]; however, research on $R$. anatipestifer is limited. In addition, there may also be major mutations in nonQRDRs $[17,18]$. Therefore, in addition to QRDRs, nonQRDRs are also worthy of attention.

Linda [19], Viktòria Làzàr [20] and Csaba Pà [21] et al. all showed that quinolone-resistant $E$. coli mutants exhibited cross-resistance to other types of antibiotics. In addition, Webber et al. [22] reported that DNA gyrase mutants of Salmonella typhimurium L821(Ser83Phe) and L825 (Asp87Gly) showed different susceptibilities to some antimicrobials, such as beta-lactams, aminoglycosides, and folate synthesis inhibitors.

In this study, we determined the minimum inhibitory concentration (MIC) values of four quinolone drugs for $162 R$. anatipestifer isolates to investigate the prevalence of quinolone resistance in $R$. anatipestifer. Then, we detected mutations in the $\operatorname{gyr} \mathrm{A}, \operatorname{parC}$ and $\operatorname{parE}$ gene fragments of the $162 R$. anatipestifer isolates and constructed $R$. anatipestifer mutants with high-frequency in vivo mutation sites to explore whether mutations at these sites contribute to the quinolone resistance mechanism of $R$. anatipestifer and to investigate whether these mutations can cause cross-resistance.

\section{Methods}

\section{R. anatipestifer strains}

A total of $162 R$. anatipestifer strains were used in this study. Among these strains, 137 clinical isolates for fluoroquinolone resistance analysis were obtained from 48 duck farms from 2013 to 2018, 15 other clinical isolates were isolated from 14 duck farms from 1998 to 2012, one strain was obtained from the American Type Culture Collection (ATCC) and 9 strains were obtained from the Culture Collection of the University of Gothenburg (CCUG). Detailed strain information is shown in Additional file 1: Table S1.

All $R$. anatipestifer isolates were cultured on $5 \%$ blood tryptic soy agar (TSA; Oxoid, Basingstoke, UK). These inoculated plates were incubated at $37^{\circ} \mathrm{C}$ in a $5 \% \mathrm{CO}_{2}$ atmosphere for $24 \mathrm{~h}$. Polymerase chain reaction (PCR) amplification of 16S rRNA was performed to identify $R$. anatipestifer as described previously [9].

\section{Minimum inhibitory concentrations (MICs) of quinolones}

MICs of nalidixic acid (Aladdin, USA), ciprofloxacin (Aladdin, USA), enrofloxacin (Aladdin, USA) and ofloxacin (Meilunbio, China) for $162 R$. anatipestifer isolates were determined using the standard microscale broth dilution method, performed according to Clinical and Laboratory Standard Institute (CLSI) criteria [23], to analyze the antimicrobial susceptibility of randomly collected isolates between 1932 and 2018.

To explore whether the $R$. anatipestifer site-directed in vivo mutants showed cross-resistance phenotypes with other nonquinolone antibiotics, the MICs of several other nonquinolone antibiotics such as ampicillin (Aladdin, USA), ceftiofur (Meilunbio, China), amikacin (Meilunbio, China), florfenicol (Aladdin, USA), doxycycline (Meilunbio, China) and lincomycin (Aladdin, USA) were determined for these strains.

The concentration of these antimicrobial agents in 96well plates ranged from 0.25 to $1024 \mu \mathrm{g} / \mathrm{mL}$, except that of nalidixic acid, which ranged from 0.5 to $2048 \mu \mathrm{g} / \mathrm{mL}$. E. coli ATCC 25922 was used for quality control. The experiments were repeated in triplicate.

\section{PCR amplification of the gyrA, parC and parE genes}

Thirty-seven complete genomes of $R$. anatipestifer strains were sequenced by our team in a previous study, and these sequences were uploaded to the National 
Center for Biotechnology Information (NCBI, http:// www.ncbi.nlm.nih.gov/genome) database. The accession numbers of these complete genomes are listed in Additional file 2: Table S2. We compared the $g y r \mathrm{~A}, g y r \mathrm{~B}$, $\operatorname{par} \mathrm{C}$, and parE genes of these thirty-seven complete genomes. The comparison results are shown in Additional file 3-6: Table S3-S6. A region with a high mutation probability that included a large number of mutations was selected as the target region for PCR amplification. The region includes more than just the QRDR. Five fragments were selected (GyrA amino acids $51-327$ and 442-726; ParC amino acids 349-615 and 610-857; and ParE amino acids 312-590). There were no eligible areas in the GyrB. The primers were designed with ATCC 11845 as the template to amplify the above five sequences. In addition to the 37 whole-genome sequencing strains, the remaining 125 clinical isolates were prepared as DNA templates by heat and lysis. The above five regions of 125 clinical isolates were amplified to obtain the mutant sites in these regions. In addition, Sanger sequencing was also carried out for the above five regions of the 37 whole-genome sequencing strains to verify the accuracy of the sequences. PCR primers are listed in Additional file 7: Table S7.

\section{Sequence alignment analysis}

The sequenced fragments of the 125 clinical isolates and the 37 complete genomes of $R$. anatipestifer strains were compared with the genome of ATCC 11845 (as a reference strain) by BLAST [24]. The genomic sequence of ATCC 11845 was downloaded from NCBI. MEGA 7 [25] was used for alignment and to obtain mutation sites in all $R$. anatipestifer strains.

\section{Construction of $R$. anatipestifer mutants in vivo}

The regions where the high-frequency mutation sites were located were selected to design primers. The target fragments of the three genes of ATCC 11845 were amplified via PCR, and the amplified fragments were ligated to a $\mathrm{Ncol} / \mathrm{XhoI}-$ digested pOES suicide vector to generate pOES-fragments (gyrA fragment-1, 514 bp; gyrA fragment-2, $944 \mathrm{bp} ;$ parC fragment-1, $454 \mathrm{bp}$; parC fragment-2, $433 \mathrm{bp}$; parE fragment, $1026 \mathrm{bp})$. Subsequently, pOES fragments were transformed into $E$. coli $\mathrm{DH} 5 \alpha$. The recombinant plasmids were extracted using the E.Z.N.A ${ }^{\circ}$ Plasmid Mini Kit I (Omega Bio-Tek, USA). The QuikChange ${ }^{\bullet}$ Lightning Site-Directed Mutagenesis Kit (Stratagene, CA) was used to construct the sitedirected mutations in recombinant plasmids. Subsequently, these recombinant plasmids containing site-directed mutations were transformed into E. coli S17-1. The transconjugants were selected on LB plates with ampicillin (Amp, $100 \mu \mathrm{g} / \mathrm{mL}$ ).

The donor strain E. coli S17-1 harboring the suicide vector pOES fragments and the recipient strain $R$. anatipestifer ATCC 11845 were grown on LB and sheep blood plates, respectively, at $37^{\circ} \mathrm{C}$ overnight. Targeted mutants were constructed by introducing recombinant DNA into the cells by conjugation [26]. The transconjugants were selected on blood agar plates supplemented with cefoxitin (Cfx, $1 \mu \mathrm{g} / \mathrm{mL}$ ) and kanamycin (Kana, $50 \mu \mathrm{g} / \mathrm{mL}$ ). Transconjugants were shaken in GC broth (GCB) at $37^{\circ} \mathrm{C}$ overnight to lose the suicide plasmid. Then, $200 \mu \mathrm{L}$ of the appropriate dilution was plated on GCB agar supplemented with $13 \mathrm{mM} \mathrm{p}-\mathrm{Cl}$-Phe to select clones without the plasmid [27]. Clones were screened by PCR using primers cfx P1/P2 to choose Cfx-sensitive colonies. Then, the $\mathrm{Cfx}$-sensitive colonies were sequenced with the corresponding primers. The primers and plasmids used in this study are shown in Table 1. Growth curves were plotted for the ATCC 11845 strain and $R$. anatipestifer mutants grown in TSB medium at $37^{\circ} \mathrm{C}$. $\mathrm{OD}_{600}$ was recorded at 2 -h intervals.

\section{Results \\ Minimum inhibitory concentrations (MICs) of nalidixic acid, ciprofloxacin, enrofloxacin, and ofloxacin}

The MICs of four antibacterial drugs were determined for a total of $162 R$. anatipestifer strains. The MIC values for all strains are shown in Additional file 1: Table S1.

Since the number of collected strains before 2013 was limited, we selected $137 R$. anatipestifer strains from 48 farms from 2013 to 2018 to analyze the prevalence of quinolone resistance (Table 1). The MIC values of four quinolone drugs for the $137 R$. anatipestifer strains were all greater than $90 \%$. A total of $100 \%(137 / 137)$ of the $R$. anatipestifer strains were resistant to nalidixic acid. Meanwhile, $R$. anatipestifer strains that showed resistance to ciprofloxacin, enrofloxacin, or ofloxacin were determined to be $97.8 \%$ (134/137), $99.3 \%(136 / 137)$ or $97.8 \%(134 / 137)$ of the total, respectively.

\section{Amino acid substitutions of GyrA, GyrB and ParC in quinolone-resistant $R$. anatipestifer}

We sequenced five gene fragments of $\operatorname{gyr} \mathrm{A}$, parC and parE of $162 R$. anatipestifer strains. The detailed amino acid substitutions in the amplified fragments are shown in Additional file 1: Table S1. In GyrA, amino acid position 83 contained two mutation types: Ser83Arg (26/162) and Ser83Ile (125/162). Moreover, the strains that were resistant to ciprofloxacin (149/ 162), enrofloxacin (151/162), and ofloxacin (149/162) all had mutations at position 83. In addition, the mutation frequencies of Cys465Arg (69/162) in GyrA; Val586Thr/Ala (67/162), Val799Ala (117/162), and Ile811Val (109/162) in ParC; and Val357lle (131/162), His358Tyr (131/162), Arg541Lys (133/162), and Asp564Lys (72/162) in ParE were all higher than 40\%; these sites were defined as high-frequency mutation 
Table 1 Antibiotic resistance phenotypes of R. anatipestifer strains collected between 2013 and 2018

\begin{tabular}{|c|c|c|c|c|c|c|c|c|c|c|c|c|}
\hline \multirow[t]{2}{*}{ Years } & \multirow[t]{2}{*}{ Antibiotics } & \multicolumn{11}{|c|}{ MICs $(\mu \mathrm{g} / \mathrm{mL})$ and proportion of strains (\%) } \\
\hline & & 0.5 & 2 & 4 & 8 & 16 & 32 & 64 & 128 & 256 & 512 & 1024 \\
\hline \multirow[t]{4}{*}{$2013 n=13$} & NA & & & & & & & & & 7.69 & 61.5 & 30.8 \\
\hline & $\mathrm{CIP}$ & 7.69 & & & & 7.69 & 84.6 & & & & & \\
\hline & ENR & 7.69 & & & & 7.69 & 7.69 & 53.8 & 23.1 & & & \\
\hline & OFX & & 7.69 & & & & 46.2 & 23.1 & 23.1 & & & \\
\hline \multirow[t]{4}{*}{$2014 \sim 2015 n=6$} & NA & & & & & & & & & 16.7 & 83.3 & \\
\hline & CIP & & & 16.7 & & 16.7 & 50 & 16.7 & & & & \\
\hline & ENR & & & 16.7 & & & 16.7 & 50 & 16.7 & & & \\
\hline & OFX & & & 16.7 & & 16.7 & 33.3 & 16.7 & 16.7 & & & \\
\hline \multirow[t]{4}{*}{$2016 n=18$} & NA & & & & & & & & & 11.1 & 77.8 & 11.1 \\
\hline & $\mathrm{CIP}$ & & & & 5.6 & 55.6 & 38.9 & & & & & \\
\hline & ENR & & & & 5.6 & 22.2 & 27.8 & 33.3 & 11.1 & & & \\
\hline & OFX & & & & 11.1 & 44.4 & 44.4 & & & & & \\
\hline \multirow[t]{4}{*}{$2017 n=60$} & NA & & & & & & & & & 10 & 42 & 8 \\
\hline & CIP & & 3.3 & 5.0 & 1.7 & 21.7 & 61.7 & 6.7 & & & & \\
\hline & ENR & & & 8.3 & 1.7 & 8.3 & 28.3 & 36.7 & 16.7 & & & \\
\hline & OFX & & 3.3 & 5.0 & 11.7 & 28.3 & 20 & 18.3 & 13.3 & & & \\
\hline \multirow[t]{4}{*}{$2018 n=40$} & NA & & & & & & & & & 17.5 & 57.5 & 25 \\
\hline & CIP & & & & 7.5 & 35 & 35 & 22.5 & & & & \\
\hline & ENR & & & & 2.5 & 50 & 10 & 12.5 & 25 & & & \\
\hline & OFX & & & & 2.5 & 47.5 & 12.5 & 22.5 & 15 & & & \\
\hline
\end{tabular}

${ }^{a} N A$ nalidixic acid; CIP ciprofloxacin; ENR enrofloxacin; OFX ofloxacin

sites. Asn202Glu (52/162) in GyrA and Ile390Thr (45/162), Gly752Val (42/162), Val768Ala (42/162), Glu827Asp (42/162), and Met833Ile (42/162) in ParC had mutation frequencies between 20 and $40 \%$; these sites were defined as medium-frequency mutation sites. Twenty-eight amino acid mutations with mutation frequencies lower than $20 \%$ were observed (the detailed mutation sites are shown in Additional file 1: Table S1).
The correspondence between different mutation types and MIC values of different drugs are shown in Additional file 8: Table S8. The number of mutants containing Ser83Ile in GyrA; Val799Ala and Ile811Val in ParC; and Val357Ile, His358Tyr, and Arg541Lys in ParE was the highest (44/162), whereas mutants with Ser83Ile in GyrA and Val357Ile, His358Tyr and Arg541Lys in ParE were the second most frequent $(21 / 162,13.0 \%)$. Compared to the ATCC 11845 strain, the strains with these

Table 2 MICs for site-directed R. anatipestifer mutants

\begin{tabular}{|c|c|c|c|c|c|c|c|c|c|c|}
\hline \multirow[t]{2}{*}{ Mutants } & \multicolumn{10}{|c|}{$\operatorname{MICs}(\mu \mathrm{g} / \mathrm{mL})^{a}$} \\
\hline & NA & $\mathrm{CIP}$ & ENR & OFX & AMP & EFT & AK & FFC & DO & MY \\
\hline ATCC 11845 & 256 & 0.25 & 0.25 & 0.25 & $<0.25$ & 1 & 64 & 2 & $<0.25$ & 0.25 \\
\hline ATCC gyrA (Ser83lle) & 512 & 2 & 4 & 4 & 64 & 128 & 128 & 2 & $<0.25$ & 0.25 \\
\hline ATCC gyrA (Cys465Arg) & 256 & 0.25 & $<0.25$ & 0.25 & 16 & 32 & 32 & 2 & $<0.25$ & $<0.25$ \\
\hline ATCC parC (Val586Ala) & 256 & 0.25 & $<0.25$ & 0.5 & 64 & 128 & 64 & 2 & $<0.25$ & $<0.25$ \\
\hline ATCC parC (lle811Val) & 256 & $<0.25$ & $<0.25$ & 0.25 & 64 & 128 & 64 & 2 & $<0.25$ & 0.25 \\
\hline ATCC parC (Val799Ala, lle811 Val) & 512 & 0.25 & 0.25 & 0.25 & 64 & 128 & 64 & 2 & $<0.25$ & 0.25 \\
\hline ATCC parE (Val357lle, His358Tyr) & 256 & 0.25 & 0.25 & 0.25 & 64 & 128 & 64 & 2 & $<0.25$ & 0.25 \\
\hline ATCC parE (Val357lle, His358Tyr, Arg541Lys) & 512 & 0.25 & 0.25 & 0.25 & 32 & 128 & 64 & 2 & $<0.25$ & 0.25 \\
\hline ATCC gyrA (Ser83lle) + parE (Val357lle, His358Tyr, Arg541Lys) & 256 & 4 & 4 & 2 & 128 & 256 & 64 & 2 & $<0.25$ & $<0.25$ \\
\hline
\end{tabular}

${ }^{a}$ NA nalidixic acid; CIP ciprofloxacin; ENR enrofloxacin; OFX ofloxacin; AMP ampicillin, EFT ceftiofur; AK amikacin; FFC florfenicol; DO doxycycline; MY lincomycin 
mutation types exhibited a 32 - to 512-fold increase in the MICs of the fluoroquinolone drugs, but the MIC value of nalidixic acid did not change significantly.

\section{Characterization of $R$. anatipestifer mutants}

Eight mutant strains, which were selected based on sites with mutation frequencies greater than $40 \%$, were constructed to explore the contribution of different mutation sites to the resistance of $R$. anatipestifer. The MIC values of four antibacterial drugs were determined for the mutants (Table 2). Regardless of the amino acid substitution, the MIC of nalidixic acid did not change significantly. The high-frequency mutations Val799Ala and Ile811Val in ParC and/or Val357Ile, His358Tyr and Arg541Lys in ParE, which were present without the amino acid 83 substitution in GyrA, had no significant effect on MIC values. Compared to the parent strain, only the mutant strains with the amino acid 83 substitution in GyrA, namely, the ATCC gyrA (Ser83Ile) and ATCC gyrA (Ser83Ile) + parE (Val357Ile, His358Tyr, Arg541Lys) strains, exhibited an 8- to 16-fold enhancement in resistance to fluoroquinolones. In contrast, the high-frequency mutation sites Cys465Arg in GyrA; Val799Ala and Ile811Val in ParC; and Val357Ile, His358Tyr and Arg541Lys in ParE had no effect on quinolone resistance.

To understand whether the site-directed mutants exhibit low sensitivity to other types of antibiotics, the MICs of six nonquinolone antibiotics were determined for these strains. The results showed that the MICs of ampicillin and ceftiofur were significantly increased for the mutants. Interestingly, regardless of which gene among gyrA, parC, and parE has amino acid substitutions, the MIC value of ampicillin and ceftiofur increased significantly.

To explore whether these site mutations would affect the adaptability of the strain, the growth curves of the site-directed mutant strains and the parent strain were plotted (Fig. 1). Compared to the parent strain, the mutants exhibited no significant difference in growth, except for the ATCC parC (Ile811Val) strain.

\section{Discussion}

In the past six years, the resistance rate of $R$. anatipestifer to four quinolones has increased. The resistance rate to nalidixic acid, which was $60 \%$ before 2003 [28] and $87.4 \%$ between 1999 and 2009 [29], has risen to $100 \%$ between 2013 and 2018. The resistance rate to ciprofloxacin increased from 23.1 to $59.2 \%$ between 1998 and 2010 [29-31]. The resistance rate to enrofloxacin was approximately $60 \%$ between 1999 and 2010 [29, 30]. Meanwhile, Zhong et al. [31] and Zhang et al. [29] determined that the resistance rate of $R$. anatipestifer isolated from different years to ofloxacin was approximately $24 \%$. Nevertheless, the resistance rate to ofloxacin increased to $51.5 \%$ between 2008 and 2010. The resistance rates of $R$. anatipestifer isolated between 2013 and 2018 to CIP, ENR and OFX were 97.8, 99.3 and 97.8\%, respectively. Compared with the resistance rate determined in the past, it can be seen that the resistance of $R$. anatipestifer to quinolones has increased. The high prevalence of fluoroquinolone resistance in $R$. anatipestifer isolates from ducks may be due to the overuse and abuse of fluoroquinolones in duck disease treatment.

The MIC of nalidixic acid is interesting. The MIC values of nalidixic acid against ATCC 11845 and CCUG 18373, which were isolated in 1932 and 1955, respectively, were both $256 \mu \mathrm{g} / \mathrm{mL}$, notwithstanding the fact that quinolones were not used in that period. Therefore, in addition to point mutations, there are other mechanisms that mediate resistance to nalidixic acid.

The emergence of mutations is consistent with the history of the development of quinolone drugs. In the 1980s, third-generation quinolone antibiotics were synthesized successively. The strains isolated from 1960 to 1980 are sensitive to FQs, while the RA-CH-2 and RCAD0392 strains isolated in the 1990s showed a resistant phenotype to FQs (Additional file 1: Table S1). The
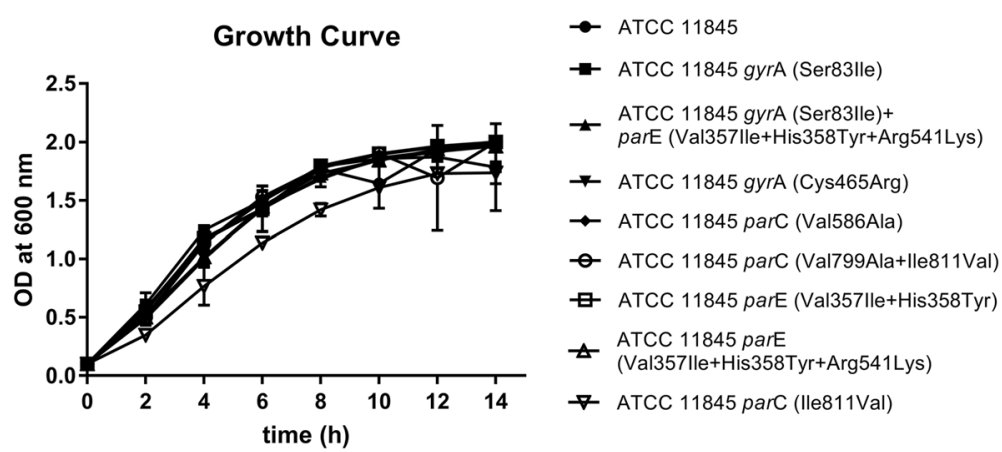

Fig. 1 Growth curves for R. anatipestifer ATCC 11845 and R. anatipestifer site-directed mutants. R. anatipestifer ATCC 11845 and site-directed mutants were cultured $\left(\mathrm{OD}_{600}=0.1\right)$ in $20 \mathrm{~mL}$ of TSB, and the growth curves were determined 
proportion of fluoroquinolone-resistant strains increased significantly. This phenomenon may be due to the increased use of FQs and/or the third-generation quinolone antibiotics being highly conducive to enriching $R$. anatipestifer resistant strains.

The presence of the amino acid 83 mutation increases the MIC value of fluoroquinolones. All fluoroquinoloneresistant isolates had a mutation at the amino acid 83 site in GyrA (Additional file 1: Table S1), and the sitedirected mutants with an amino acid 83 mutation showed an 8- to 16-fold increase in MIC values. These results indicate that the amino acid 83 mutation in GyrA is critical to confer fluoroquinolone resistance to $R$. anatipestifer. This finding is consistent with previous research results $[32,33]$. There are two mutant types at the amino acid 83 position in GyrA, Ser83Arg and Ser83Ile. Previous reports suggest that the mutation type Ser83Ile provides more resistance to FQs than Ser83Arg [29]. However, we found that several strains containing the Ser83Arg mutation type have FQ MICs ranging from 64 to $128 \mu \mathrm{g} / \mathrm{mL}$. Both Ser83Ile and Ser83Arg can confer low-level or high-level resistance to strains, and Ser83Arg can also confer sensitivity to strains. The same type of mutation also has a variety of base substitution methods. The Ser83Ile group contains AGC-ATC and AGC-ATT mutations, while the Ser83Arg group contains AGC-AGA and AGC-CGC mutations. The same mutant types with different base substitutions had difference in resistance to FQs. For strains with Ser83Arg, some of which had the AGC-AGA base substitution type, the MICs of FQs were $4 \mu \mathrm{g} / \mathrm{mL}$ or less; the strains in which the base substitution type was AGC-CGC had MIC values of FQs greater than or equal to $8 \mu \mathrm{g} / \mathrm{mL}$. The reason for this phenomenon remains to be explored.

It is strange that while more than $90 \%$ of $R$. anatipestifer strains have high-frequency mutations in parC and parE, these mutations have no significant effect on fluoroquinolone resistance. Perhaps these types of highfrequency mutations are highly adaptive to the evolution of the strain [12, 34]; however, the exact significance of these mutations remains to be explored. Interestingly, although these high-frequency mutations have no significant effect on fluoroquinolone resistance, they all increase the resistance of strains to betalactams. This result is consistent with previous reports. The reason for the change in the sensitivity to nonquinolones is probably due to altered patterns of supercoiling and hence global expression of stress response pathways [20,22].

The RCAD0133 strain has 22 mutations, including all high-frequency mutations; however, this strain has a low level of resistance to fluoroquinolone drugs. These results suggest that not all mutations confer resistance to fluoroquinolones [35] and that the number of mutations is not related to the level of fluoroquinolone resistance.

The substitutions Asp87His in GyrA and Arg120Glu in ParC, which might be associated with fluoroquinolone resistance, were reported by Sun et al. [5]. Additionally, Zhang et al. [29] suggested that Gly81Asp in GyrA might play a sufficient role in enrofloxacin resistance. However, none of these three mutation sites were detected in this test. The Glu202Asn mutation in GyrA [29] and mutations at positions 357 and 358 in ParE, which may increase the resistance of RA to fluoroquinolones, were reported. These mutation sites are consistent with the detected mutations in this study, but the MIC results for the site-directed mutants showed that amino acids 357 and 358 had no significant effect on fluoroquinolone resistance. ATCC parC (Val799Ala, Ile811Val) grew significantly better than ATCC parC (Ile811Val) in the same media conditions (Fig. 1). This phenomenon may be due to the generation of compensatory mutations that increase the adaptability of the strain [36].

There are many strains that have the same mutation type but have different drug resistance phenotypes, such as RCAD0354 and RCAD0356, but these strains exhibit a 2- to 8-fold difference in MICs of FQs. This phenomenon indicates that there may be other mechanisms, such as other low-frequency mutations, undetected sites or efflux pumps.

\section{Conclusions}

The resistance rate of $R$. anatipestifer to four quinolones is considerably high in the duck industry. Sequencing results and the MIC of site-directed mutants showed that only the strains containing the amino acid 83 mutation exhibited resistance to FQs; the other high-frequency mutation sites did not significantly contribute to the MICs. This study highlights the importance of using fluoroquinolones reasonably and correctly to reduce the emergence of multidrug-resistant strains; moreover, it provides data for the molecular detection of fluoroquinolone-resistant $R$. anatipestifer strains.

\section{Supplementary information}

Supplementary information accompanies this paper at https://doi.org/10. 1186/s12866-019-1659-4

Additional file 1: Table S1. Information on the mutation sites in the gyrA, parC and parE genes of $R$. anatipestifer

Additional file 2: Table S2. Origin and accession numbers of 37 whole-genome sequences of $R$. anatipestifer

Additional file 3: Table S3. Amino acid sequence alignment of GyrA from the 37 whole-genome-sequenced $R$. anatipestifer strains

Additional file 4: Table S4. Amino acid sequence alignment of GyrB from the 37 whole-genome-sequenced $R$. anatipestifer strains 
Additional file 5: Table S5. Amino acid sequence alignment of ParC from the 37 whole-genome-sequenced $R$. anatipestifer strains

Additional file 6: Table S6. Amino acid sequence alignment of ParE from the 37 whole-genome-sequenced $R$. anatipestifer strains

Additional file 7: Table S7. Primers and plasmids used in this study

Additional file 8: Table S8. Antibiotic phenotype and amino acid substitutions in GyrA, ParC and ParE of R. anatipestifer.

\section{Abbreviations}

ATCC: American Type Culture Collection; CCUG: Culture Collection of the University of Gothenburg; CLSI: Clinical and Laboratory Standards Institute; MDR: multidrug resistance; MIC: minimum inhibitory concentration; NCBI: National Center for Biotechnology Information; PCR: polymerase chain reaction; PMQR: plasmid-mediated quinolone resistance; QPDRs: quinoloneresistance-determining regions; TSA: tryptone soy agar; TSB: tryptone soy broth

\section{Acknowledgements}

Not applicable.

\section{Authors' contributions}

$D Z, M Z$ and $A C$ conceived and designed the project. $M Z, J H, L Z, Y L$ and $M L$ determined the MICs for the $R$. anatipestifer isolates. MZ, XC, LP and $Y Y$ performed PCR amplification of the gyrA, parC and parE genes. MZ, QY, YW, $\mathrm{SZ}$ and $\mathrm{XZ}$ constructed the $R$. anatipestifer mutants in vitro and characterized the mutants. JX, MW, RJ and SC performed sequence alignment analysis. MZ and DZ drafted and revised the manuscript. All authors have read and approved the final version of the manuscript.

\section{Funding}

This work was supported by the National Key Research and Development Program of China under grant no. 2017YFD050080; China Agricultural Research System under grant no. CARS-42-17; Sichuan Veterinary Medicine and Drug Innovation Group of China Agricultural Research System under grant no. CARS-SVDIP; International S\&T Cooperation Program of Sichuan Province under grant no. 2017HH0026; and Science and Technology Innovation Program of Guizhou Academy of Agricultural Science under grant no. (2017)03. The funders had no role in study design and collection, analysis, and interpretation of data, or preparation of the manuscript.

\section{Availability of data and materials}

The data supporting the results of this paper are available in the NCBI WGS database. The accession numbers of $R$. anatipestifer genomes are included in Additional file 2: Table S2.

\section{Ethics approval and consent to participate}

The Animal Ethics Committee of the Sichuan Agricultural University waived the need for ethics approval and the need to obtain informed consent for the sample collection.

\section{Consent for publication}

Not applicable.

\section{Competing interests}

The authors declare no conflicts of interest.

\section{Author details}

${ }^{1}$ Research Center of Avian Diseases, College of Veterinary Medicine, Sichuan Agricultural University, Chengdu, Sichuan, China. ${ }^{2}$ Key Laboratory of Animal Disease and Human Health of Sichuan Province, Chengdu, Sichuan, China. ${ }^{3}$ Institute of Preventive Veterinary Medicine, Sichuan Agricultural University, Chengdu, Sichuan, China. ${ }^{4}$ Guizhou Animal Husbandry and Veterinary Research Institute, Guiyang, Guizhou, China.
Received: 11 May 2019 Accepted: 22 November 2019

Published online: 03 December 2019

\section{References}

1. Ruiz JA, Sandhu TS. Riemerella anatipestifer infection. In: Swayne DE, Glisson $J R$, McDougald LR, Nolan LK, Suarez DL, Nair VL, editors. Diseases of poultry, 13th edition. Hoboken: Wiley; 2013. p. 823-8.

2. Andersson MI, MacGowan AP. Development of the quinolones. J Antimicrob Chemother. 2003;51(Suppl. S1):1-11.

3. Endtz HP, Ruijs GJ, van Klingeren $B$, Jansen WH, van der Reyden T, Mouton RP. Quinolone resistance in campylobacter isolated from man and poultry following the introduction of fluoroquinolones in veterinary medicine. J Antimicrob Chemother. 1991;27(2):199-208.

4. Nhung NT, Chansiripornchai N, Carrique-Mas JJ. Antimicrobial resistance in bacterial poultry pathogens: a review. Front Vet Sci. 2017:4:126.

5. Sun N, Liu J-H, Yang F, Lin D-C, Li G-H, Chen Z-L, Zeng Z-L, et al. Molecular characterization of the antimicrobial resistance of Riemerella anatipestifer isolated from ducks. Vet Microbiol. 2012;158(3-4):376-83.

6. Chen Y-P, Tsao M-Y, Lee S-H, Chou C-H, Tsai H-J. Prevalence and molecular characterization of chloramphenicol resistance in Riemerella anatipestifer isolated from ducks and geese in Taiwan. Avian Pathol. 2010;39(5):333-8.

7. Chen YP, Lee $\mathrm{SH}$, Chou CH, Tsai HJ. Detection of florfenicol resistance genes in Riemerella anatipestifer isolated from ducks and geese. Vet Microbiol. 2012;154(3-4):325-31.

8. Yang F-F, Sun Y-N, Li J-X, Wang H, Zhao M-J, Su J, Zhang Z-J, Liu H-J, Jiang $\mathrm{S}-J$. Detection of aminoglycoside resistance genes in Riemerella anatipestifer isolated from ducks. Vet Microbiol. 2012;158(3-4):451.

9. Luo H, Liu M, Wang L, Zhou W, Wang M, Cheng A, Jia R, Chen S, Sun K, Yang $\mathrm{Q}$, et al. Identification of ribosomal RNA methyltransferase gene ermF in Riemerella anatipestifer. Avian Pathol. 2015;44(3):162-8.

10. Luo HY, Liu MF, Wang MS, Zhao XX, Jia RY, Chen S, Sun KF, Yang Q, Wu Y, Chen $X Y$, et al. A novel resistance gene, Inu(H), confers resistance to lincosamides in Riemerella anatipestifer CH-2. Int J Antimicrob Agents. 2017.

11. Zhu D-K, Luo H-Y, Liu M-F, Zhao X-X, Jia R-Y, Chen S, Sun K-F, Yang Q, Wu $Y$, Chen $X-Y$. Various profiles of tet genes addition to tet $(X)$ in Riemerella anatipestifer isolates from ducks in China. Front Microbiol. 2018;9:585.

12. Redgrave LS, Sutton SB, Webber MA, Piddock LJ. Fluoroquinolone resistance: mechanisms, impact on bacteria, and role in evolutionary success. Trends Microbiol. 2014:22(8):438-45.

13. Khodursky AB, Cozzarelli NR. The mechanism of inhibition of topoisomerase IV by quinolone antibacterials. J Biol Chem. 1998;273(42):27668-77.

14. Ren YN, Zhen PP, Jian LI, Guo YF, Wang LH, Ren JY, et al. Genetic characterization of highly fluoroquinolone-resistant clinical Escherichia coli strains from China: role of QRDR,acrR,marR and soxR. China Anim Husbandry Vet Med. 2012;39:11-6.

15. Singhal R, Reynolds PR, Marola JL, Epperson LE, Arora J, Sarin R, Myneedu VP, Strong M, Salfinger M. Sequence analysis of fluoroquinolone resistanceassociated genes gyrA and gyrB in clinical Mycobacterium tuberculosis isolates from patients suspected of having multidrug-resistant tuberculosis in New Delhi, India. J Clin Microbiol. 2016:54(9):2298-305.

16. Qin $T, B i$, Fan W, Kang $H$, Ma $P$, Gu B. Novel mutations in quinolone resistance-determining regions of gyr $\mathrm{A}$, gyrB, parC and parE in Shigella flexneri clinical isolates from eastern Chinese populations between 2001 and 2011. Eur J Clin Microbiol Infect Dis. 2016:35(12):2037-45.

17. Al-Emran HM, Heisig A, Dekker D, Adu-Sarkodie Y, Cruz Espinoza LM, Panzner U, von Kalckreuth V, Marks F, Park SE, Sarpong NJ. Detection of a novel gyrB mutation associated with fluoroquinolone-nonsusceptible Salmonella enterica serovar Typhimurium isolated from a bloodstream infection in Ghana. Clin Infect Dis. 2016;62(suppl_1):S47-9.

18. Nawaz M, Sung K, Kweon O, Khan S, Nawaz S, Steele R. Characterisation of novel mutations involved in quinolone resistance in Escherichia coli isolated from imported shrimp. Int J Antimicrob Agents. 2015;45(5):471-6.

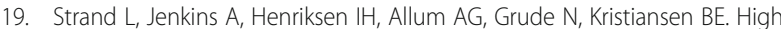
levels of multiresistance in quinolone resistant urinary tract isolates of Escherichia coli from Norway; a non clonal phenomen? BMC Res Notes. 2014;7(1):376

20. Lázár V, Nagy I, Spohn R, Csörgő B, Györkei Á, Nyerges Á, Horváth B, Vörös A, Busa-Fekete R, Hrtyan M. Genome-wide analysis captures the determinants of the antibiotic cross-resistance interaction network. Nat Commun. 2014:5:4352. 
21. Pál C, Papp B, Lázár V. Collateral sensitivity of antibiotic-resistant microbes. Trends Microbiol. 2015;23(7):401-7.

22. Webber MA, Ricci V, Whitehead R, Patel M, Fookes M, Ivens A, Piddock LJ. Clinically relevant mutant DNA gyrase alters supercoiling, changes the transcriptome, and confers multidrug resistance. MBio. 2013;4(4):e00273-13.

23. Clinical and Laboratory Standards Institute (CLSI). Performance Standards for Antimicrobial Susceptibility Testing: Twenty-seventh Informational Supplement. CLSI document M100-S27. Wayne: Clinical and Laboratory Standards Institute; 2017.

24. Altschul SF, Gish W, Miller W, Myers EW, Lipman DJ. Basic local alignment search tool. J Mol Biol. 1990;215(3):403-10.

25. Kumar S, Stecher G, Tamura K. MEGA7: Molecular Evolutionary Genetics Analysis version 7.0 for bigger datasets. Mol Biol Evol. 2016;33(7):1870

26. Liao H, Cheng X, Zhu D, Wang M, Jia R, Chen S, Chen X, Biville F, Liu M, Cheng A. TonB energy transduction systems of Riemerella anatipestifer are required for iron and hemin utilization. PLoS One. 2015;10(5):e0127506.

27. Liu M, Huang Y, Liu J, Biville F, Zhu D, Wang M, Jia R, Chen S, Zhao X, Yang $Q$, et al. Multiple genetic tools for editing the genome of Riemerella anatipestifer using a counterselectable marker. App Microbiol Biotechnol. 2018;102(17):7475-88.

28. Chang C-F, Lin W-H, Yeh T-M, Chiang T-S, Chang Y-F. Antimicrobial susceptibility of Riemerella anatipestifer isolated from ducks and the efficacy of ceftiofur treatment. J Vet Diagn Investig. 2003;15(1):26-9.

29. Zhang CZ. Antimicrobial susceptibility of Riemerella anatipestifer isolates from ducks and geese and the mutations of Gyrase associated with quinolone resistance. J Vet Med Taiwan. 2012;38(1):7-18.

30. Gyuris É, Wehmann E, Czeibert K, Magyar T. Antimicrobial susceptibility of Riemerella anatipestifer strains isolated from geese and ducks in Hungary. Acta Vet Hung. 2017;65(2):153-65.

31. Zhong CY, Cheng AC, Wang MS, Zhu DK, Luo QH, De Zhong C, Li L, Duan Z. Antibiotic susceptibility of Riemerella anatipestifer field isolates. Avian Dis. 2009;53(4):601-7.

32. Hopkins KL, Davies RH, Threlfall EJ. Mechanisms of quinolone resistance in Escherichia coli and Salmonella: recent developments. Int J Antimicrob Agents. 2005;25(5):358-73.

33. Yonezawa M, Takahata M, Banzawa N, Matsubara N, Watanabe Y, Narita H. Analysis of the NH2-terminal 83rd amino acid of Escherichia coli GyrA in quinolone-resistance. Microbiol Immunol. 1995;39(4):243-7.

34. N W MJE. The emergence of antibiotic resistance by mutation. Clin Microbiol Infect. 2007;13(1):5-18.

35. Santhosh KS, Deekshit VK, Venugopal MN, Karunasagar I, Karunasagar I. Multiple antimicrobial resistance and novel point mutation in Fluoroquinolone-resistant Escherichia coli isolates from Mangalore, India. Microb Drug Resist. 2017;23(8):994-1001.

36. Melnyk AH, Wong A, Kassen R. The fitness costs of antibiotic resistance mutations. Evol Appl. 2015;8(3):273-83.

\section{Publisher's Note}

Springer Nature remains neutral with regard to jurisdictional claims in published maps and institutional affiliations.

Ready to submit your research? Choose BMC and benefit from:

- fast, convenient online submission

- thorough peer review by experienced researchers in your field

- rapid publication on acceptance

- support for research data, including large and complex data types

- gold Open Access which fosters wider collaboration and increased citations

- maximum visibility for your research: over $100 \mathrm{M}$ website views per year

At $\mathrm{BMC}$, research is always in progress.

Learn more biomedcentral.com/submissions 